\title{
«Pour instruire leurs filles » : du dépaysement au renouveau dans les écrits de Marie de l'Incarnation
}

\author{
Judith Sribnai \\ Université de Montréal
}

Retraçant une "généalogie du racisme », Elsa Dorlin évoque «La nouvelle division de la Terre par les différentes races d'hommes qui l'habitent », texte de François Bernier paru en 1684 dans le Journal des Sçavans où le terme de " race » prendrait, pour la première fois en français, son acception moderne (210). Au $\mathrm{XVI}^{\mathrm{e}}$ et jusqu'au début du XVII ${ }^{\mathrm{e}}$ siècle, la « race » postule bien " une différence transmise par la naissance » et l'importance "de traits de caractère hérités » (Boulle 159). Mais, il arrive que la qualité du sang dégénère et, plus rarement, elle s'acquiert (Jouanna 157 et sqq.) : être bien-né relève d'un effort, d'une éducation, de l'apprentissage d'une façon de vivre, de manger, de bouger, d'un savoir-faire social qui sont autant de manières de n'être pas, ou plus, bourgeois ou paysans ${ }^{1}$. Avec le texte de Bernier, le terme renverrait à " une détermination endogène, un principe de discrimination qui transcende les familles, mais aussi les frontières politiques et culturelles de la terre, rendu nécessaire en raison de l'intensification des migrations » (Dorlin 211).

La typologie de Bernier compte « quatre ou cinq Especes ou Races » (133). Quatre ou cinq, car le cas des Américains reste un peu flou : "Pour ce qui est des Americains, ils sont à la verité la plûpart olivastres, \& ont le visage tourné d'une autre maniere que nous, néanmoins je n'y trouve point une assez grande difference pour en faire une espece particuliere $\&$ differente de la nostre » (136). D'autre teint, d'autre manière et, cependant, pas si différents : cette ambivalence rappelle la lecture que Tzvetan Todorov propose de l'expérience de Colomb en Amérique. Colomb voulait les Américains semblables à lui, désirait ardemment les rendre même. Et pourtant, il ne les voit pas : «Colon a découvert l'Amérique, mais non les Américains» si bien que «toute l'histoire de la découverte de l'Amérique, premier épisode de la conquête, est frappée de cette ambiguïté : l'altérité humaine est à la fois révélée et refusée» (56). Le regard sur les Américains est pris dans la violence de cette alternative : être assimilé et devenir même, ou ne pas être reconnu.

Entre l'arrivée des Espagnols en Amérique du Sud et la publication de la classification de Bernier, la France commence d'occuper l'Amérique du Nord. À côté des enjeux stratégiques et économiques de la colonisation, des politiques

\footnotetext{
${ }^{1}$ Sur le lignage et la pureté du sang comme modèle de discrimination, voir Schaub et Sabastiani.
} 
d'assimilation des populations autochtones sont mises en œuvre, portées par ce que Georges Sioui appelle le "mythe de la disparition de l'autochtone » (2; Delâge, Le pays renversé; Dickason). Les entreprises d'évangélisation du premier $\mathrm{XVII}^{\mathrm{e}}$ siècle y jouent un rôle central où résonne toujours cette rencontre biaisée de l'altérité analysée par Todorov. Dans un texte paru en 2009, le théologien Jacques Racine reformulait ainsi le problème que soulève, pour plusieurs chercheurs et chercheuses, l'histoire jésuite en Nouvelle France: «comment concilier une véritable reconnaissance de l'autre et l'appel à la conversion? » (297). Racine cherche, quant à lui, une réponse théologique qui puisse, malgré les conséquences de cette histoire, sauver la vocation missionnaire de l'Église.

Je propose de revenir ici sur cette question dont la charge philosophique me paraît essentielle et qui dit les impasses d'un rapport à l'autre qui, si l'on hésite à le qualifier théoriquement de raciste ${ }^{2}$, n'en est pas moins gros d'une idée de force et de mort. Pour cela, je m'intéresserais aux œuvres de Marie Guyart, dite de l'Incarnation, fondatrice des Ursulines à Québec, arrivée en Nouvelle France en 1639. Figure incontournable de l'Amérique française, tant d'un point de vue historique (Deslandres), mystique (Nadeau-Lacour), pédagogique (Gourdeau) que religieux (Bremond), elle est aussi, quoiqu'il y ait remarquablement peu d'études à ce sujet, une écrivaine. Elle raconta, dans des lettres et des Relations, l'histoire, pleine de douleurs et d'extases, de sa rencontre avec Dieu et de la vocation extraordinaire qui la conduisit jusqu'aux "grandes vastitudes" (R 130) ${ }^{3}$, cette terre de la " Mission de Canada » (R 133), " pays des Sauvages » (R 135). Elle évoque ainsi leur première rencontre, au sortir du navire :

Nous fîmes rencontre de plusieurs Sauvages, abordant aux terres : ce qui nous apporta une grande joie. Ces pauvres gens n'ayant jamais vu personnes faites comme nous, étaient tous dans l'admiration, et, lorsqu'on leur dit que nous étions filles de Capitaines qui, pour l'amour d'eux, avions quitté notre pays, nos parents et tous les délices, ils étaient ravis d'étonnement, et encore plus, que c'étaient pour instruire leurs filles, à ce qu'elles ne fussent pas brûlées dans les feux, mais pour leur enseigner comme il fallait être éternellement bienheureux. Ils ne pouvaient comprendre cela. (R 166-167)4

Portés par cette vocation pédagogique et apostolique, les écrits de Marie de l'Incarnation témoignent de cette difficile, peut-être impossible, conciliation : entre un désir (devoir) de christianiser, se confondant avec une nécessité

${ }^{2}$ En plus des raisons historiques mentionnées, on peut citer la définition proposée par Colette Guillaumin: cette "croyance en l'hétérogénéité absolue de l'autre » qui se manifeste « principalement par l'affirmation (implicite ou explicite) de l'essence de l'autre » et qui « tend à conserver la différence de l'autre en tant que groupe tout en niant la surgence de l'individu au sein du groupe. » (112)

${ }^{3}$ Pour la Relation de 1654, j'utilise l'édition d'Alessandra Ferraro (notée R suivi du folio).

${ }^{4}$ Pour les correspondances, j'utilise l'édition de Dom Guy Oury (notée C suivi du folio). Dans son introduction, Dom Guy Oury présente les difficultés d'établissement de ces lettres dont beaucoup ont été remaniées et réécrites par le fils de Marie de l'Incarnation, Claude Martin. 
" d'instruire », c'est-à-dire d'éduquer, de changer l'autre, et la rencontre de ceux ou celles que la différence (spirituelle, morale, sociale), ne rend pas moins dignes d'amour. En m'appuyant sur la Relation de 1654 et sur la correspondance de Marie de l'Incarnation, j'aimerais analyser la façon dont l'écriture de l'Ursuline nomme et construit cette difficulté, mais la façon aussi dont cette difficulté inquiète autant qu'elle informe l'épreuve du discours. Un tel exercice de lecture, qui fait place aux imaginaires et aux récits dans lesquels Marie de l'Incarnation a puisé ses représentations du "Sauvage », permettra peut-être de réfléchir à la façon dont on peut aborder ce corpus, si bouleversant à bien des égards, sans faire silence sur les malaises que suscitent la description de ces «pauvres gens » dont il faut instruire les filles. La paresse consisterait à faire de Marie de l'Incarnation une écrivaine de son temps. Mais, précisément, qu'a-t-elle fait des récits, des représentations, des discours de son temps?

Au fil de ses écrits, se développe une pensée de l'éducation, des espoirs et des limites de la francisation des jeunes Autochtones, jeunes filles d'abord rêvées, puis prises dans les stries d'une géographie politique, spirituelle, quotidienne, où Marie de l'Incarnation cherche, encore et toujours, à discerner les signes d'un renouveau - ce pénible et mystérieux geste de devenir autre tout en se découvrant toujours même.

\section{«Si bien dressés à la Françoise » : christianiser, franciser, dépayser}

Avant le départ en Nouvelle France, il y eut, pour Marie de l'Incarnation, au moins deux « rencontres » avec les peuples du Canada. À « l'âge de trente-quatre à trente-cinq ans », alors qu'elle vient tout juste de prononcer ses vœux aux Ursulines de Tours, l'Esprit du Christ « s'empara », dit-elle, de son esprit «pour qu'il n'eût plus de vie que dans le sien et par le sien » (R 129). Ce jour-là, « cet Esprit [la] portait en esprit dans les Indes, au Japon, dans l'Amérique, dans l'Orient, dans l'Occident, dans les parties du Canada et dans les Hurons » (R 130). Se promenant «dans ces grandes vastitudes », elle y voit des millions de " pauvres âmes » pour lesquelles elle demande le salut. Au récit de ces « vues et certitudes » (R 130), le père Dinet évoque la mission canadienne à la jeune femme qui, jusqu'alors, n'avait " jamais su qu'il y eût un Canada au monde » (R 133). De ce point de vue, l'autobiographie mystique est, forcément, le récit d'une altérité, celle, première, du « divin Époux » bien sûr, mais celle aussi de ces peuples jamais vus, d'abord indifférenciés, en tout cas ignorants de la révélation. Et, de même que le chemin de la foi consiste à sans cesse retrouver en soi un Dieu qui était déjà là, Marie de l'Incarnation part trouver ces «Sauvages » que, déjà, elle porte en elle :

Il me semblait que je connaissais toutes les âmes rachetées du Sang du Fils de Dieu, en quelque coin de la terre habitable qu'elles pussent être, et mon amour se portait à celles 
qui étaient les plus abandonnées dans les pays des Sauvages où je me promenais sans cesse. (R 134).

Durant les cinq années qui suivent, Marie de l'Incarnation défend fermement ce dessein que plusieurs, y compris chez les Jésuites, prennent pour «fantaisies » (R 142, 146-147) $)^{5}$ et dont l'exécution est en partie tenue secrète (L 75). À partir de là, elle prend connaissance des Relations, recueil des lettres rapportant les expéditions des pères de la Compagnie de Jésus en Nouvelle France, en plus d'échanger avec plusieurs missionnaires (R 137). C'est la deuxième " rencontre » avec des populations dont les contours, au fil des lectures et des conversations, se précisent, plus seulement «Sauvages », mais Hurons ou Iroquois, avec un " pays », Canada ou Huronie, tantôt « heureux païs » de conversion (L 56) ou « païs tres mauvais » de froid et de dureté (L 34) et, surtout, des jeunes filles pour lesquelles l'ursuline souhaite fonder un couvent (L 64). L'autre, d'abord signe divin, devient celui qu'ont raconté et publié des voyageurs dont l'hérö̈sme et le dévouement séduisent. Avant donc de faire «rencontre avec plusieurs Sauvages » en "abordant aux terres », Marie de l'Incarnation est porteuse des représentations et des imaginaires diffusés et en partie élaborés par les écrits jésuites et dont les critiques ont bien montré les difficultés et les enjeux tant rhétoriques (Laflèche; Ouellet; Le Bras) que politiques ou moraux (Greer; Paschoud; Reichler; Tinguely). Et ces âmes qu'elle semble connaître, elle fera en sorte de les rendre reconnaissables : telles qu'elle les a imaginées, telles qu'elle les espère.

Les œuvres de Marie de l'Incarnation participent donc d'un contexte de conquête politique et spirituelle qui se lit, par exemple, dans l'incessant comptage des âmes, recueillies, amenées au couvent, parfois sauvées : "l'on nous amena un grand nombre de filles Sauvages pour les disposer au saint baptême dans le Séminaire; [...] on en baptisa cinq à la fois » (L 159), «Dieu amène des Sauvages de tous côtez, pour les faire enroller au nombre de ses enfans » (L 197), « on parle de nous donner deux filles de cette nation avec deux Algonquines, outre dix-huit dont notre Séminaire a été rempli, sans parler des filles externes qui y viennent continuellement»(L 95). Tout se chiffre et s'inventorie, les baptêmes, comme le pain et les dépenses quotidiennes, chaque augmentation est une réussite et se raconte parce qu'elle témoigne d'une colonie qui prospère :

Si cette paix dure [avec les Iroquois et les Anglais], comme il y a lieu de l'espérer, ce païs sera très-bon et très-commode pour l'établissement des François, qui se multiplient beaucoup et font assez bien leurs affaires par la culture des terres qui deviennent bonnes à présent que l'on abat les grandes forests qui la rendent si froide. [...] Cette paix

5 Au père Lejeune, elle affirme par exemple en 1636 : «Je vous diray que si telle est la volonté de Dieu, qu'il n'y a rien en ce monde qui m'en puisse empescher, quand mesme je devrois estre engloutie des ondes en chemin » (L 60) 
augmente le commerce, particulièrement des Castors [...]. Mais le trafic des âmes est le contentement de ceux qui ont passé les mers pour les venir chercher, afin de les gagner à Jésus-Christ. L'on en espère une grande moisson par l'ouverture des Hiroquois. (L 545)

Faire «trafic », c'est échanger, commercer, faire circuler ${ }^{6}$. Comme les biens, les âmes passent d'une culture à l'autre, d'un monde païen au monde chrétien, passages qui sont faits eux aussi, de négociations et de discussions, d'un certain calcul d'intérêt, dessinant de nouveaux sillons sur le territoire. Abattre les forêts pour faire des lignes de semis, engager un commerce, vendre des fourrures, semer la parole sainte et récolter «moisson » d'âmes ${ }^{7}$ : c'est un seul et même souffle. Santé économique, politique et spirituelle se nouent autour de l'espérance d'une récolte prospère qui suppose à la fois de faire de la place (abattre les forêts) et d'occuper (l'« établissement des François »). La conversion des âmes dépend d'une telle transaction des êtres et des cœurs (le «contentement» des nouveaux arrivants) qu'elle manifeste en même temps: certaines âmes prennent la mer et s'exilent, passant d'Europe en Amérique, pour en « venir chercher » d'autres, c'est-à-dire mener ces âmes ailleurs. Tout se passe comme si, en miroir de leur propre exode, les Européens contraignaient les Autochtones à quitter, eux aussi, un territoire (de forêt, de chasse, de castors) pour habiter un autre lieu (de Français, d'Anglais, de commerce), comme si les Français étaient venus «chercher» les Hurons et les Iroquois pour les amener en Nouvelle France. Ce dépaysement forcé est aussi bien géographique qu'intérieur.

La formation des jeunes filles aux Ursulines de Québec obéit en effet aux mêmes règles qu'en France où l'éducation religieuse est inséparable d'une instruction morale et culturelle (Gourdeau : 49 et sqq.) ${ }^{8}$. Pour être converties (conduites à la révélation), les jeunes Autochtones sont également menées dans un autre espace physique, précisément dans une autre France bâtie et reproduite

${ }^{6}$ Richelet donne par exemple : «Ce mot se dit en parlant de marchands et de marchandises et veut dire commerce, négoce » et « C'est à dire, faire commerce de réputation, rendre loüange pour loüange. Donner de la réputation à ceux qui nous en donnent, donner encens pour encens ». Furetière, quant à lui, rattache le terme à l'histoire des colonies et au statut social : «Vente, ou eschange de marchandises, de billets, d'argent en commerce, et à negotier. Le trafic est interdit en France aux Gentilshommes. Les Nobles Venitiens et Genois font le trafic en gros. C'est un vilain trafic que celuy des esclaves, des Benefices. Il entend bien le trafic. C'est un trafic inconnu. Les Hollandois veulent faire tous seuls le trafic des Indes. »

${ }^{7}$ Parmi les paraboles de la semence, on peut rappeler celle que l'on trouve dans 3 Rois 18 : le prophète Elie engage une compétition avec les prophètes de Baal pour savoir lequel du dieu profane ou du chrétien répondra aux prières de ses fidèles. C'est Elie qui gagne bien sûr. L'image de la moisson et de la récolte est liée à l'idée de la reconnaissance du vrai Dieu.

${ }^{8}$ L'éducation religieuse dispensée par les Ursulines s'adressent aussi bien aux Françaises de la colonie qu'aux Autochtones (L 112). 
au sein du cloître. Ainsi, évoquant toujours la paix avec les Iroquois, l'ursuline raconte la venue d'une "capitainesse avec sa compagnie», Iroquoise appartenant aux délégations de paix. Elle explique le travail de séduction qui s'engage : "Nous les avons régalez deux fois splendidement à leur mode, car c'est ainsi qu'il les faut attirer » (565) et ajoute : «Ils ont pris un singulier plaisir à voir et à entendre nos Séminaristes, et entr'autres une petite Huronne de dix à onze ans que nous Francisons. Elle sçait lire, écrire et chanter en trois langues, sçavoir en Latin, en François et en Huron » (id). Marie Aouentohon, en somme, dépayse la délégation iroquoise qui admire cette enfant francisée, dont la langue fait entendre un autre pays (la France), une autre culture (le latin) ${ }^{9}$. Truchement d'exotisme et annonce publicitaire, elle manifeste tous ces gestes par lesquels les jeunes Autochtones en sont venues à vivre un nouveau pays, rendues « mêmes » pour les Ursulines, mais bizarrement lointaines pour la délégation iroquoise. Je donnerai deux exemples de ces pratiques de dépaysement, toutes deux liés à l'épreuve du corps : le nettoyage et la sédentarisation ${ }^{10}$.

Marie de l'Incarnation évoque à quelques reprises «la saleté des filles sauvages qui n'étaient point encore faites à la propreté des Français » et qui « faisait trouver un soulier en notre pot, et journellement des cheveux et des charbons» (R 173). Elles apportent avec elles une «salleté insupportable » et, raconte l'ursuline dans une lettre de septembre 1640 :

Quand on nous les donne elles sont nues comme un ver, et il les faut laver depuis la tête jusqu'aux pieds, à cause de la graisse dont leurs parens les oignent par tout le corps : et quelque diligence qu'on fasse, et quoi qu'on les change souvent de linge et d'habits, on ne peut de long-temps les épuiser de la vermine causée par l'abondance de leurs graisses. Une Sœur employe une partie du jour à cela. (L 97)

La graisse, qui n'a plus fonction de protection, est nid de « vermine », proximité avec une nature grouillante et certainement aussi, pour les Sœurs, souvenir de la misère des gueux de France. S’il faut enlever la couche de graisse, il faut, en même temps, cacher la nudité des séminaristes, autrement dit, littéralement, les nettoyer d'une histoire et les revêtir d'une autre, ce que Claire Gourdeau résume ainsi :

De plus, aux yeux de Marie de l'Incarnation, l'importance de vêtir ses séminaristes à la française ne tend pas seulement à les protéger du froid ou à améliorer leurs conditions hygiéniques, mais vise également à transformer l'apparence extérieure de ses pupilles, à rendre visible les signes de la civilisation française que les religieuses européennes sont venues porter au Canada. (Gourdeau 65)

${ }^{9}$ La jeune fille d'ailleurs, porte-parole de la politique française, témoigne « le plaisir qu'elle avoit de la paix » (L 565).

10 Il y aurait beaucoup d'autres exemples de la façon dont, dans les écrits de Marie de l'Incarnation, sont mis en récit les gestes qui visent à effacer ou transformer la culture des jeunes filles qui passent par les Ursulines : la langue, on le voit, mais également le changement de nom, l'apprentissage du français, les vêtements, le mariage. 
On peut également ajouter à ce geste celui d'effacer la trace des parents, ceux qui, justement, ont enduit de graisse le corps des enfants. Dans cette même lettre adressée à une bienfaitrice, Marie de l'Incarnation fait le portrait de ses séminaristes, de leur foi, de leur douceur. Elle conclut en disant : «Je serois trop longue à vous parler séparément de toutes, mais je vous diray en général que ces jeunes filles nous aiment plus que leur parens, ne témoignant aucun desir de les suivre, ce qui est fort extraordinaire dans les Sauvages » (L 96). Elle ajoute qu'après les exercices spirituels, « on ne peut exprimer les caresses qu'elles nous firent, ce qu'elles ne font jamais à leurs mères naturelles. » (L 97). D'une mère à l'autre, les séminaristes changent de lieu, sont «francisées", c'est-à-dire débarrassée de la sauvagerie (la nudité, la vermine, le gras animal), pour être comme les petites Françaises, vêtues, témoignant leur attachement à leurs mères spirituelles, préférant l'âme à la nature.

Dans la même lettre, l'ursuline conte l'histoire de la première séminariste qu'on donna au couvent, Marie Negabmat :

La première séminariste Sauvage qu’on nous donna appelée Marie Negabmat étoit si accoutumée à courir dans le bois que l'on perdoit toute espérance de la retenir dans le Séminaire. Le R. Père Le Jeune qui avoit porté son Père à nous la donner, envoya avec elle deux grandes filles Sauvages Chrétiennes qui demeurèrent quelque temps avec elle pour la fixer; mais ce fut en vain, car elle s'enfuit quatre jours après dans les bois aiant mis en pièces une robe que nous lui avions donnée. (L 95)

Son père, « excellent chrétien », la ramène au séminaire où elle « ne fut pas deux jours qu'il y eut un changement admirable ». Portée par la prière et la piété, « elle ne sembloit plus être elle-même ». Devenue «l'exemple des filles de Québec», Marie Negabmat ne s'enfuit plus elle est «fixée ». N'être plus soi-même par la grâce de la conversion, c'est une expérience intérieure que Marie de l'Incarnation connaît bien, j'y reviendrai. Ce que j'aimerais souligner ici, c'est la nécessité, pour convertir, de retenir et d'obliger à une autre expérience géographique. Si le geste et la pratique du croyant sont toujours création ou resémantisation des espaces (de Certeau, La faiblesse de croire, 31 et sqq.), s'ajoutent dans ce cas plusieurs nouvelles frontières : celles qui fondent la vie conventuelle (la clôture ou l'espace des Ursulines contre l'ouverture des bois); celles qui distinguent des manières d'habiter (sédentaire contre nomade ou semi-nomade); celles qui marquent les genres (les jeunes filles bien élevées ne courent pas dans les bois). L'enfermement est, dans la correspondance de Marie de l'Incarnation, le signe d'une victoire de la grâce. Dans une lettre de 1640 à l'un de ses frères, elle vante ces « filles sauvages qui n'ont plus rien de la barbarie » et qui peuvent « demeurer enfermées dans un cloître », et elle célèbre ailleurs les «Sauvages sédentaires », si zélés, à l'image des premiers chrétiens (L 119). En effet, les jeunes filles «perdent ce qu'elles ont de sauvage si tôt qu'elles sont lavées des eaux du saint 
baptême en sorte que ceux qui les ont vues auparavant courir dans les bois comme des bêtes sont ravis et pleurent de joye de les voir douces comme des brebis s'approcher de la sainte table pour y recevoir le véritable agneau » (L 112). Perdant "ce qu'elles ont de sauvage », ce ne sont plus des «bêtes ", mais des brebis, animaux domestiqués, un temps égarés peut-être, mais qui sauront reconnaître l'agneau, et rejoindre ainsi un bestiaire biblique et une signification emblématique familiers des Sœurs.

Dans ces exemples, l'appel à la conversion ne saurait être la reconnaissance de l'autre. Conduire les jeunes filles au Royaume de Dieu, c'était aussi les amener au Royaume de France et, en ce sens, il s'agissait, pour les Ursulines, d'en faire des semblables, de trouver, sous la saleté grattée, un air connu ${ }^{11}$.

Mais la civilité et la politesse européennes sont-elles si nécessaires? Marie de l'Incarnation exalte très souvent dans ses écrits l'extraordinaire piété de ces filles «dans le vray chemain du ciel » (L 137) et, si elle les compare aux Françaises, celles d'Europe ou celles de la Nouvelle France, c'est toujours à l'avantage des premières : «je n'eusse jamais creu que des filles nées dans la barbarie eussent pris des plis comme les filles de France, et très avantageusement » (L 137); les enfants « ont de si grandes inclinations à la pureté » (L 146); elle se confessent régulièrement, avouent leurs fautes, leur ferveur éblouit les Pères jésuites de passage (L 147); elles apprennent les louanges de Dieu si vite que, dit l'ursuline, « je n'ay jamais veu des filles Françoises les dispositions que je remarque en ellesmêmes » (L 103) $)^{12}$. Les jeunes Autochtones sont jugées fort pieuses, mais aussi, plus souvent qu'autrement, ayant des mœurs plus sages que les Françaises, surtout celles qui vivent en Nouvelle France :

L'on est fort soigneux en ce païs de faire instruire les filles Françoises; et je vous puis assurer que s'il n'y avoit des Ursulines elles seroient dans un danger continuel de leur salut. [...] Enfin ce que je puis dire est que les filles en païs sont pour la pluspart plus sçavantes en plusieurs matières dangereuses, que celles de France. (L 802)

\footnotetext{
${ }^{11}$ Marie de l'Incarnation relaie, sur ce point, les efforts politiques de la Compagnie de Jésus. Ainsi écrit-elle à son fils en 1654 : « Nous avons des Huronnes que les Révérends Pères ont jugé à propos que nous élevassions à la Françoise : car comme tous les Hurons sont à présent convertis, et qu'ils habitent proches des François, on croit qu'avec le temps ils pourront s'allier ensemble, ce qui ne se pourra faire que les filles soient francisées tant de langue que de mœurs. » (L 545-546). Qui est ce « on » qui parle ici ? On comprend en tout cas que la «francisation », dont sont en charge les Ursulines, s'inscrit dans un projet politique de plus grande envergure.

12 Également, à l'un de ses frères : «L'on nous figuroit le Canada comme un lieu d'horreur : on nous disoit que c'étoit les faubourgs de l'Enfer, et qu'il n'y avoit pas au monde au pays plus méprisable. Nous expérimentons le contraire, car nous y trouvons un Paradis que pour mon particulier je suis indigne d'habiter. Il y a des filles sauvages qui n'ont rien de la barbarie. » (L 112) et «Nous voyons souvent de semblable ferveur dans nos bons Néophites, qui sans mentir font honte à ceux qui sont nez de parens Chrétiens » (L 201).
} 
À l'inverse, Marie de l'Incarnation ne cache pas son admiration devant les « bons coups » des Algonquins pour répondre aux attaques iroquoises :

Une femme Algonquine aiant été enlevée par les Hiroquois avec toute sa famille, son mari qui étoit étroitement lié de toutes parts, lui dit que si elle vouloit elles les pouvoit sauver tous. Elle entendit bien ce que cela vouloit dire, c'est pourquoi elle prit sont temps pour se saisir d'une hache, et avec un courage non pareil elle fend la tête au Capitaine, coupe le col à un autre, et fit tellement la furieuse qu'elle mit tous les autres en fuite : Elle délie son mari et les enfants et retirent tous sans aucun mal en un lieu d'assurance. (L 563)

À la lecture de cette anecdote, on peut s'étonner que l'auteure salue les coups de hache donnés par la furieuse Algonquine. On est loin, après tout, des bonnes mœurs enseignées aux séminaristes ou aux pensionnaires. En réalité, ces brouillages sont fréquents : on peut être pieuse de cœur et « sauvage » de culture; les Françaises peuvent devenir plus féroces et canailles que les «Sauvages » et les «Sauvages » plus louables que les Françaises; une femme peut tuer à coups de hache et faire figure de dévotion. S’il est préférable, pour arriver au Royaume de Dieu, de passer par celui de France, il n'en reste pas moins que toute anthropologie, toutes formes de politique ou de projet colonial sont dépassées par un universalisme chrétien qui, en définitive, rend caduques toutes distinctions culturelles ou ethniques - mais non spirituelles.

\section{«On est sauvage toute l'année »}

Dans la Relation de 1654, Marie de l'Incarnation raconte comment il lui fallut se «mettre à l'étude de la langue des Sauvages $»^{13}$. Parce qu'il y a plus de vingt ans qu'elle n'avait "raisonner sur aucune chose qui tînt de la science et spéculation », la tâche lui parut insurmontable :

[...] d'abord cette étude d'une langue si disproportionnée à la nôtre, me fit bien mal à la tête, et me semblait, qu'apprenant des mots par cœur et les verbes, - car nous étudions par préceptes, - que des pierres me roulaient dans la tête, et puis des réflexions sur une langue barbare! tout cela me faisait croire qu'humainement je n'y pouvais réussir. (R 170)

Pourtant l'expérience d'une étrangeté d'abord irréductible se résorbe :

J'en traitais amoureusement avec Notre-Seigneur, lequel m'aida en sorte qu'en peu de temps j'y eus une très grande facilité, en sorte que mon occupation intérieure n'en était point ni empêchée ni interrompue. Mon étude était une oraison qui me rendait suave cette langue qui ne m'était plus barbare. J'en sus assez en peu de temps pour pouvoir instruire nos chers néophytes en tout ce qui était requis en leur salut. (id.)

${ }^{13}$ Elle apprend d'abord l'algonquin et le montagnais, elle apprendra ensuite le huron et puis, à 50 ans, l'iroquois. Elle écrit notamment une histoire sacrée en algonquin, un dictionnaire et catéchisme en iroquois, un dictionnaire en algonquin (L 801). 
Ce passage est important, car il manifeste et explique une géographie du monde qui repose moins sur l'origine, l'ethnie, la langue ou les mœurs, que sur l'appartenance ou non au christianisme. Ainsi l'étude de la langue devient oraison, prière où les mots « barbares » sont finalement entendus et compris par Dieu. Il n'y a plus alors de «barbare ", c'est-à-dire plus d'étrangers, mais la perpétuation d'un colloque continue avec le «divin Époux», suture inespérée des langues et des peuples, éloignés depuis l'épisode de Babel, souvenir également du miracle de la Pentecôte et d'une parole messianique pour le salut universel. Dans une lettre à l'une des religieuses de la Visitation de Tours, Marie de l'Incarnation évoque à nouveau ces «épines à apprendre un langage si contraire au nôtre ». On se moque d'elle pourtant, car « on [lui] représente que si la peine étoit si grande, [elle] n’y auroi[t] pas tant de facilité ». Elle explique alors : «Mais croyez-moy, le désir de parler fait beaucoup : je voudrois faire sortir mon cœur par ma langue pour dire à mes chers Néophites ce qu'il sent de l'amour de Dieu et de Jésus notre bon maître » (L 125). La diversité des cœurs et des langages s'épuise ainsi dans un amour qui ne connait pas de borne. À la même religieuse à qui elle adressait quelques phrases en "langue sauvage », elle dit : «Voilà qui m’est échapé. C'est à dire en notre langue : Ma sœur encore que vous soyez bien loin...» (L 108). Dès 1640, et quoiqu'elle en trouve l'apprentissage fort difficile jusqu'à ses dernières années, la «langue sauvage» est «notre » langue, sa langue et celle de la communauté qui gravite autour du couvent.

Ceci explique plusieurs éléments qui semblent entrer en tension ou en contradiction avec le travail de francisation évoqué plus haut. De même que les Français peuvent devenir "Sauvages », de même Marie de l'Incarnation admet rapidement que, comme la langue, la vie « sauvage » est devenue sienne. En «ce bout de monde ", dit-elle à l'un de ses frères, «l'on est sauvage toute l'année, sinon lorsque les vaisseaux sont arrivez que nous reprenons notre langue Françoise » (L 102). Dans ce cas, le terme « sauvage » désigne à la fois une façon de vivre où se mêle l'étrangeté d'une existence inconnue en France, l'expérience d'un quotidien où se rencontrent des habitudes et des gestes différents, et, pour les Ursulines, la joie d'une épreuve et d'une misère qui confirment la vertu de leur sacerdoce. Ces dernières pensaient en effet n'avoir pour tout logis qu'une « cabane d'écorce » (L 98), elles ont finalement une petite maison « si pauvre que nous voions par le plancher reluire les estoiles durant la nuit, et qu'à peine y peut-on tenir une chandelle allumée à cause du vent» (id.), dans laquelle elles vivent avec les jeunes Autochtones, séminaristes, pensionnaires ou celles que Marie de l'Incarnation appelle les « oyseaux passagers » (L 802). Elles reçoivent au couvent les familles, leur offrent des "festins» (L 113) et, vivant dans un grand dénuement, "très-pauvres », elles accueillent les "affamez », ceux qui fuient les conflits avec les Iroquois (L 304). Les écrits de l'Ursuline évoquent 
peu la vie commune, mais, étant donné l'exiguité des lieux, la misère quotidienne, et passé le dégoût que suscite «quelque morceau de boucan» (L 126), ils laissent penser que, dans l'enceinte du cloître, il existe une proximité relative : les Ursulines et les Autochtones partagent leurs langues, les repas, l'usure de la pauvreté, les maladies et, pour certains, une conviction spirituelle, ils commercent aussi, s'entraident (L 287), se soutiennent. De ce point de vue, on ne discerne ni la Correspondance ni dans la Relation de 1654 de peur de « passer pour des Sauvages » (Delâge, «L'influence des Amérindiens » 50).

On l'a dit, le baptême rend la «sauvagerie » positive : "Les Sauvages sont naturellement ingrats, comme nous l'expérimentons en ceux qui ne sont pas baptisez, mais pour ceux qui le sont, la grâce dont leurs âmes sont embellies les rend tres-reconnoissans $[. .]$.$» (L 166). Ils appartiennent désormais à un monde$ de piété, fait de rituels, de chants, de démonstrations, de signes, de géographies que Marie de l'Incarnation reconnait ou décrypte. Ils peuvent, eux aussi, partir évangéliser (L 544), et sont des sujets d'édification. Précisément, c'est au sein de cette ressemblance spirituelle que l'ursuline admet, et parfois défend ${ }^{14}$, une différence culturelle qu'à force elle ne cherche plus à gommer. C'est là, à mon sens, que l'appel à la conversion, paradoxalement, empêche autant qu'il rend possible la reconnaissance de l'autre : les âmes devenues si belles par le baptême n'en sont pas moins « sauvages ».

Alors que Louis XIV, par l'intermédiaire de Jean Talon, tente d'encourager le développement démographique, industriel et commercial de la colonie, Marie de l'Incarnation avoue à son fils en septembre1668:

C'est pourtant une chose très difficile, pour ne pas dire impossible de les franciser ou civiliser. Nous en avons l'expérience plus que tout autre, et nous avons remarqué de cent de celles qui ont passé par nos mains à peine en avons nous civilisé une. Nous y trouvons de la docilité et de l'esprit, mais lors qu'on y pense le moins elles montent par dessus notre clôture et s'en vont courir dans les bois avec leurs parens, où elles trouvent plus de plaisir que dans tous les agrémens de nos maisons Françoises. (L 809)

De même, en octobre de la même année :

Je ne sçai à quoi tout cela se terminera, car pour vous parler franchement, cela me paroît très-difficile. Depuis tant d'années que nous sommes établies en ce païs, nous n'en avons pu civiliser que sept ou huit, qui aient été francisées; les autres qui sont en grand nombre, sont toutes retournées chez leurs parens, quoi que très-bonnes Chrétiennes. (L 828)

Le succès très limité de la francisation et de la conversion des Autochtones (Dickason, The Myth of the Savage 260; Delâge 187 et sqq.) s'explique : "la vie sauvage leur est si charmante à cause de la liberté, que c'est un miracle de les

14 Voir par exemple sur le statut du chant dans les Constitutions L 653. 
pouvoir captiver aux façons d'agir des François qu'ils estiment indignes d'eux, qui font gloire de ne point travailler qu'à la chasse ou à la navigation » (id.). Pour comprendre que l'on puisse être « très-bonnes chrétiennes » sans être francisées, Marie de l'Incarnation mobilise une pensée anthropologique et médicale : il y a des «natures » humaines, et comme elles, des natures ou «caractères » des nations qui supposent une relative fixité des êtres et des choses (Van Delft). C'est pourquoi les jeunes filles, pourtant si douces, si dociles, passent par-dessus les clôtures dès que les Sœurs ont le dos tourné :

L'humeur sauvage est faite de la sorte : elles ne peuvent être contraintes, si elles le sont, elles deviennent mélancholiques, et la mélancholie les fait malades. D’ailleurs les Sauvages aiment extraordinairement leurs enfans, et quand ils sçavent qu'ils sont tristes ils passent par-dessus toute considération pour les r'avoir, et il les faut rendre. Nous avons eu des Huronnes, des Algonquines, des Hiroquoises; celles-cy sont les plus jolies et les plus dociles de toutes: Je ne sçay pas si elles seront plus capables d'être civilisées que les autres, ni si elles retiendront la politesse Françoise dans laquelle on les élève. Je n'attens pas cela d'elles, car elles sont Sauvages, et cela suffit pour ne le pas espérer. (L 809)

L'excès de mélancolie rend « malade » et manifeste un déséquilibre des humeurs. Il est significatif que Marie de l'Incarnation choisisse un vocabulaire propre à la théorie humorale et qui, par ailleurs, réintroduit le lien familial, prévalant cette fois sur les considérations de dévotion. Les Autochtones s'insèrent dans la grande carte du monde, carte médicale, anthropologique et métaphysique où se déploie un ensemble de signes ordonnés et intelligibles aux yeux de l'ursuline. Au sein de cette cosmographie classique, Iroquois, Hurons, Algonquins ont une place et la possibilité de leur conversion au christianisme subsume et permet finalement toutes différences humaines - caractère, humeur, usages du corps, usages domestiques ou politiques. D'ailleurs, on l'a vu, ces différences sont fragiles, les séparations poreuses. Si bien que ne pas être «civilisées » ou ne pas retenir la «politesse» française, ne relève plus nécessairement d’une détermination axiologique ou d'un échec. À ce moment-là, Marie de l'Incarnation semble reconnaitre l'existence de singularités qui ne sont pas essentiellement irréductibles (après tout, les Ursulines parlent et vivent comme les «Sauvages »), pas non plus à réduire (« je n'attens pas cela d'elles ») et qui n'empêchent pas la reconnaissance d'une ressemblance (ainsi nous avons tous des humeurs et l'excès mélancolique, partout, rend malade). Pendant plusieurs années, les Iroquois apparaissent dans la correspondance comme des "barbares », féroces et dangereux, sans pitié (L 167-168; L 223; L 323 sqq., passim), et Marie de l'Incarnation reprend dans ses lettres les récits terrifiants que rapportent les Jésuites. Mais, quand la paix revient, et même sans être baptisées, les Iroquoises, comme les Huronnes, comme les Algonquines, peuvent être « jolies », « dociles », chrétiennes sans être moins « sauvages ».

\section{« Mourir à tout » : le récit du renouveau}


La pensée de l'autre se décline donc en plusieurs récits, politiques, anthropologiques, médicaux, spirituels, qui parfois entrent en conflit, parfois se complètent, dont certains supposent un rapport de force et de domination, d'autres ménagent l'espace de singularités acceptées. On ne peut pas, me semblet-il, délier ces récits les uns des autres. Enfin, s'il n'y a pas d'« hétérogénéité absolue de l'autre ", ni finalement d'espérance de faire des jeunes Autochtones des Françaises, les écrits de Marie de l'Incarnation témoignent malgré tout d'une double impasse dans le regard porté sur les habitants du Canada, paradis et pays d'épines.

La première impasse est, pourrait-on dire, narrative autant que spirituelle. La « férocité » des nations iroquoises, si souvent condamnée dans les lettres, est indispensable au récit spectaculaire et édifiant des martyrs de la Nouvelle France. En septembre 1640, Marie de l'Incarnation écrit à la Supérieure des Ursulines de Tours : «[...] trois canots ont été pris des Hiroquois. Si cela se trouve véritable [le R. P. Poncet] est pris infailliblement, et peut-être déjà mangé. Nous aurons possible un Martyr en sa personne, ce qui fera une grande jalousie des autres qui soupirent incessamment après cette haute grâce » (L 118, L 218). Elle relate également les souffrances exemplaires du père Jogues, «qui soupiroit après ce bonheur pour achever son martire » (L 208), et qui mourut avec un autre saint martyr canadien, Jean de la Lande, "trois fois martyr » et honoré comme tel, car "il a été massacré en détestation de notre sainte Foi, et de la prière que ces perfides [les Iroquois] prennent pour des sortilèges et enchantements » (L 324). Les détails lugubres ornent le récit des persécutions des «Ouvriers de l'Evangile » : coups de bâton, têtes tranchées, doigts coupés, corps brûlés ou exposés sur des pieux, chaque souffrance de la chair est une élection, le témoignage d'une foi irréductible, la possibilité d'un lien des hommes au divin. Dieu intervient in extremis et convertit les persécuteurs (L 338). Le «martyre est doux » et Marie de l'Incarnation, comme les autres, soupire après ces afflictions qui signalent, sans plus d'ambiguité, l'accomplissement de sa mission (L 33). Le fils ainsi souhaite cette expérience à sa mère qui craint, cependant, que ses " péchez [la] priveront de ce bien » et la mère dit au fils : «Si on me venoit dire : "Vostre fils est martir", je panse que j'an mourrois de joie » (L 133). Il est impossible ici de détailler l'imaginaire martyrologique de ces textes, mais, on le voit, le récit est aussi, à l'image de celui des jésuites, l'épopée sanglante et magnifique de chrétiens forcément persécutés, forcément sauvés (Pioffet). Les nations iroquoises, résistantes et alliées des Anglais, sont a priori, cet ennemi qui doit être cruel et terrifiant (Laflèche). Le récit hagiographique des chrétiens, auquel Marie de l'Incarnation participe, piège cet autre qu'on ne peut ni tout à fait voir ni tout à fait comprendre. 
La seconde impasse relève de l'expérience mystique de Marie de l'Incarnation. Il est vrai, comme le souligne Dominique Deslandres ("Mysticisme et apprentissage »), que celle-ci dispose à une reconnaissance inédite de l'autre (Hadot). La Relation de 1654 est bien le récit d'un épuisement de soi dans l'Autre, le « chaste Époux » (R 183), l'expérience intime que «l'âme a vie en Lui et de Lui », corps, âme, esprit et langue étant tous possédés, échos d'un «langage intérieur» (R 94) qui est la parole d'un autre (de Certeau). L'énonciation au « je », toujours prise dans et par la parole de l'Esprit ${ }^{15}$, rappelle l'altérité absolument constitutive de chacun : «Ce fut par des touches divines et des pénétrations de lui en moi et d'une façon admirable de retours réciproques de moi en lui, de sorte que n'étant plus moi, je demeurai lui par intimité d'amour et d'union, de manière qu'étant perdue à moi-même, je ne me voyais plus, étant devenue lui par participation » (R 77). De ce point de vue, les mystiques ont une compréhension beaucoup plus fondamentale et essentielle de l'altérité que bien d'autres philosophes jusqu'à aujourd'hui.

Tout au long de ses écrits, Marie de l'Incarnation, soucieuse de sa conversion, revient à plusieurs reprises sur la nécessité ou le désir du renouveau : « Pour bien goûter la vocation du Canada, il faut de nécessité mourir à tout; et si l'âme ne s'efforce de le faire, Dieu le faut luy-même, et se rend inexorable à la nature, pour la réduire à cette mort, qui par une espèce de nécessité l'élève à une sainteté imminente » (L 141). Chaque moment de vie est cet effort renouvelé, parfois source de ravissement, parfois de supplice, pour mourir, se débarrasser de l'ancien et renaitre à neuf : «Car enfin, il en faut venir là, et il ne faut pas penser de pouvoir vivre dans cette nouvelle terre de bénédiction qu'avec un esprit nouveau. De-là vous pouvez juger combien il y a à travailler dans une créature envieillie dans ses fautes habituelles comme je suis » (id.). Mais si les tribulations de la convertie sont si pénibles c'est que, sa vie durant, elle ne peut pas se défaire de cette nature "envieillie », tachée par ses péchés, ceux de ses pères, empesée dans une humanité faite d'histoires, de mémoires, de vices :

[...] je confesse que je suis le néant et l'impuissance même, capable de mettre des millions d'obstacles à ses signalées faveurs, et le sentiment que j’ai de moi dans la possession de sa divine familiarité et de ses magnifiques largesses dans mon âme, me tient au-delà de l'étonnement, car, de vérité, je suis une grande pécheresse, qui ai des lâchetés sans nombre, des puérilités et des faiblesses indicibles, et c'est ce qui est digne de grande admiration qu'un Dieu qui a des milliers de millions d'âmes aimantes veuille jeter les yeux sur la dernière de ses créatures [...]. (R 229)

Comme dans le cas des martyrs, le sens de l'épreuve est dans la répétition, car chaque recommencement manifeste la gloire de Dieu (Foucault) et à la peur

15 Un exemple parmi ceux qui parsèment les écrits de Marie de l'Incarnation. À propos de l'amour qui lie l'âme au Bien-Aimé : «Je ne pensais pas écrire ceci ; mais l'Esprit m’a portée là. » (R 70) 
d'être perdue succède, encore et encore, l'« étonnement» d'avoir été trouvée. Le désir du renouveau, le devoir de «mourir à tout » c'est également, et de façon indissociable, la découverte qu'on ne change pas, que les péchés, les « lâchetés », " puérilités » et «faiblesses indicibles » reviennent sans cesse, que nous sommes toujours guettés par nos « fautes habituelles».

Je m'en tiendrai à une hypothèse, mais il me semble que cette résistance du même (le soi « envieilli »), désiré autre (renouvelé, converti, sauvé) est aussi ce qui tourmente la rencontre avec les Autochtones qu'il faut convertir. Marie de l'Incarnation les appelle des « Néophytes ». Étymologiquement, ce terme, usuel dans le vocabulaire religieux, désigne ce qui est nouvellement planté. On rêve les séminaristes comme des boutures, dépaysées, plantées à neuf, dans une nouvelle terre, ce « nouveau pays », la « Nouvelle France ». Mais là aussi quelque chose résiste qui se lit dans la concurrence des noms (Nouvelle France, Canada, Huronie), dans la permanence des gestes (passer par-dessus les clôtures et courir dans le bois), dans les liens affectifs (les jeunes filles montrent leurs attachements aux Sœurs, mais sont toujours le souci de leurs parents), dans le plurilinguisme. Aussi Marie de l'Incarnation fait-elle peut-être, à l'égard de la conversion des jeunes Autochtones, la même expérience qu'à l'égard de sa propre conversion : l'impossibilité, sur terre du moins, d'être tout à fait autre, l'obligation de faire avec le même, ce qui était déjà-là et qui est moins une affaire de subjectivité pour l'auteure (un «moi» singulier) qu'une question d'histoire, de culture, d'habitudes, de liens familiaux, de gestes hérités. Ces « Sauvages » pourtant bons chrétiens constituent, en somme, le même mystère et le même paradoxe que le sujet mystique qui, malgré le renouveau, se rencontre toujours le même.

Les écrits de Marie de l'Incarnation sont un exemple de la façon dont se sont constitués des discours sur l'autre dans un contexte de colonisation et d'évangélisation, discours travaillés par des imaginaires politiques, médicaux, religieux qui, chacun, porte une compréhension distincte de ce que pouvait signifier l'occupation de l'Amérique du Nord par les Européens. Les œuvres de l'ursuline élaborent une réflexion singulière, entre reconnaissance d'une différence qui échappe et désir de transformer l'autre, d'en faire un autre justement, le même que soi; entre espoir de renouveau et sentiment d'une rémanence de l'ancien. Cette difficulté innerve toute sa trajectoire littéraire et mystique. La saisir dans ses détails, ses possibles et ses pièges, c'est aussi comprendre les conflits intellectuels et spirituels d'une figure par ailleurs majeure dans l'histoire et les représentations du Québec aujourd'hui. 


\section{Bibliographie}

Bernier, François. « La nouvelle division de la Terre par les différentes races d'hommes qui l'habitent. » Journal des savants, 24 Apr. 1684, pp. 135-139.

Boulle, Pierre H. «La construction du concept de race dans la France d'Ancien Régime. » Outre-mer, t. 89, no. 336-337, pp.155-175.

Bremond, Henri. Sainte Marie de l'Incarnation. Éditions du Cerf, 2016.

De Certeau, Michel. La faiblesse de croire. Seuil, 1987.

---. La fable mystique 1. XVT-XVII siècle. Gallimard,

Delâge, Denys. Le pays renversé : Amérindiens et Européens en Amérique du Nord. Boréal. 1991.

---. «L'influence des Amérindiens sur les Canadiens et les Français au temps de la Nouvelle France. » Lekton, vol. 2, no. 2, 1992, pp. 103-191.

Deslandres, Dominique. Croire et faire croire : les missionnaires français an XVII siècle. Fayard, 2003.

---. «Mysticisme et apprentissage des langues amérindiennes. » Cap-auxDiamants, no. 118, 2014, pp. 14-17.

Dickason, Olive Patricia. The Myth of the Savage: The Beginnings of French Colonialism in the Americas. The University of Alberta Press, 1997.

---. Les Premières nations du Canada. Septentrion, 1996.

Dorlin, Elsa. La matrice de la race. Généalogie sexuelle et coloniale de la Nation française. La Découverte. 2009.

Foucault, Michel. Du gouvernement des vivants. EHESS, 1984.

Furetière, Antoine. Dictionnaire universel contenant generalement tous les mots francais. 1690. Slatkine Reprints, 1970.

Gourdeau, Claire. Les délices de nos ccurs. Marie de l'Incarnation et ses pensionnaires amérindiennes 1639-1672. Septentrion, 1994.

Greer, Allan. The Jesuit Relations: Natives and Missionaries in Seventeenth-Century North America. Bedford, 2010.

Guillaumin, Colette. L'idéologie raciste. Genèse et langage actuel. Gallimard, 2002.

Hadot, Pierre. Exercices spirituels et philosophie antique. Albin Michel, 2003.

Jouanna, Arlette. L'idée de race en France au XVI au début du XVII siècle. Université Paul Valéry, 1981.

Laflèche, Guy. Les Saints Martyrs Canadiens. Vol. 1, Singulier, 1988.

Le Bras, Yvon. L'Amérindien dans les Relations du père Paul Lejeune (1632-1641). Les Editions de la Huit, 1994.

Marie de l'Incarnation. Correspondances. Abbaye Saint-Pierre, 1971.

Marie de l'Incarnation. Relation de 1654. Boréal, 2016.

Nadeau-Lacour, Thérèse. Une femme mystique au ccur de l'histoire. Artège Éditions, 2015.

Paschoud, Adrien. "Aborder les Relations jésuites de la Nouvelle France (16321672). » Arborescences, no. 2, 2012. 
Pioffet, Marie-Christine. La tentation de l'épopée dans les Relations jésuites. Septentrion, 1997.

Racine, Jacques. «Comment concilier la reconnaissance de l'autre et l'appel à la conversion? » Lecture inédite de la modernité aux origines de la Nouvelle-France, Edited by Raymond Brodeur et. al., Presses de l’Université Laval, 2009, pp. 297-307.

Reichler, Claude. «Littérature et anthropologie. De la représentation à l'interaction dans une Relation de la Nouvelle France au XVII ${ }^{\mathrm{e}}$ siècle. » L'Homme, no. 164, 2002, pp. 37-56.

Richelet, Pierre. Dictionnaire francois, contenant les mots et les choses, plusieurs nouvelles remarques sur la langue francoise : ses expressions propres, figurees \& burlesques. Jean-Herman Widerhold, 1680.

Schaub, Jean-Frédéric et Sebastiani, Silvia. «Savoirs de l'autre? L'émergence des questions de race. » Histoire des sciences et des savoirs, edited by Stéphane Van Damme, vol. 1, Seuil, 2015, pp. 283-304.

Sioui, Georges, Pour une histoire amérindienne de l'Amérique. Presses de l'Université Laval, 1999.

Tinguely, Frédéric. «État présent. Écritures du voyage à la Renaissance. » French Studies, no. 44, 2010, pp. 329-335.

Van Delft, Louis, Littérature et anthropologie. Nature bumaine et caractère à l'âge classique. Puf, 1993. 